\title{
Engineering a genetically encoded magnetic protein
}

\section{crystal}

Thomas L. Li $i^{1,2}$, Zegao Wang ${ }^{3}$, He You ${ }^{4}$, Qunxiang Ong ${ }^{1}$, Vamsi J. Varanasi ${ }^{1}$, Mingdong Dong ${ }^{5}$, Bai Lư ${ }^{4}$ Sergiu P. Pașca², Bianxiao Cui ${ }^{* 1}$

1. Department of Chemistry, Stanford University, Stanford, CA 94305, USA

2. Department of Psychiatry and Behavioral Sciences, Stanford University, Stanford, CA 94305, USA

3. College of Materials Science and Engineering, Sichuan University, Chengdu 610065, China

4. School of Pharmaceutical Sciences, Tsinghua University, Beijing 100084, China

5. Interdisciplinary Nanoscience Center, Aarhus University, Aarhus 8000, Denmark

Bianxiao Cui: (650) 725-9573, bcui@stanford.edu 
a

ftn-PAK4 : inka-PAK4 Mass Ratio

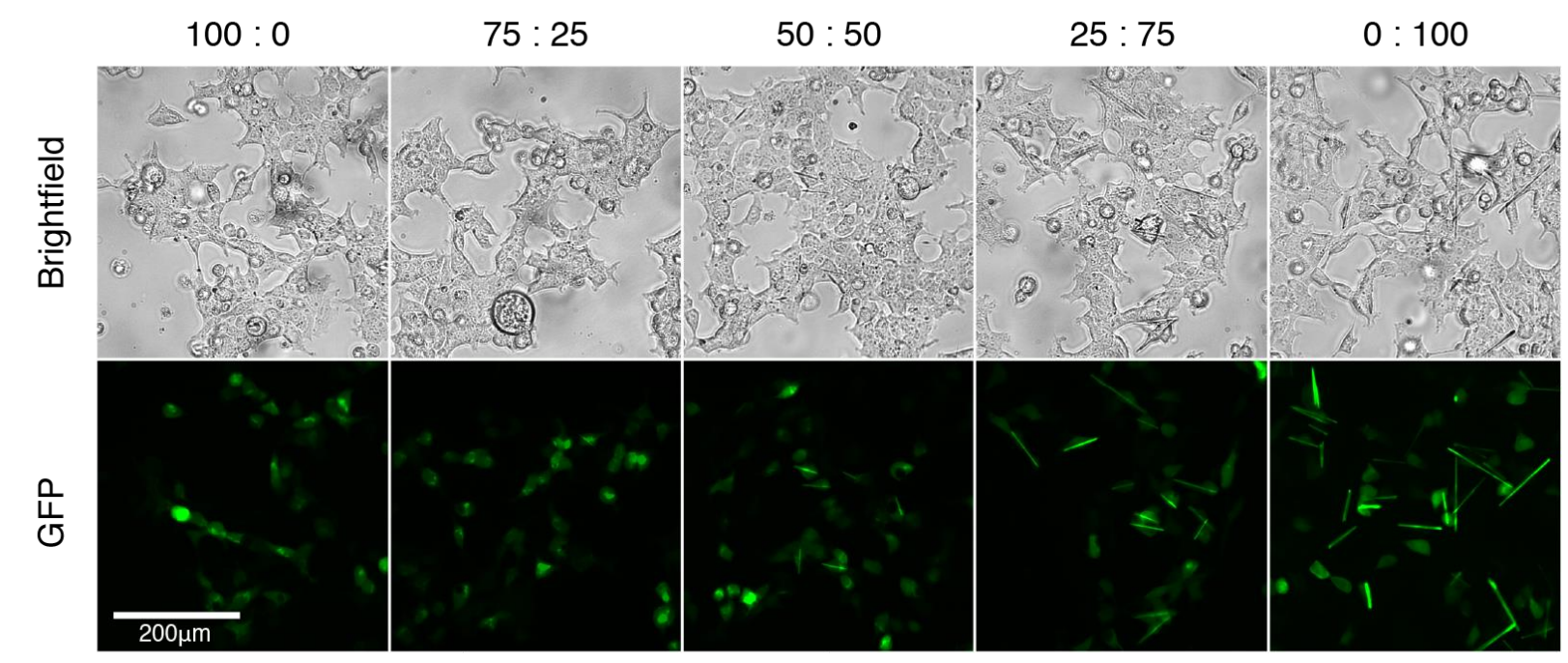

b

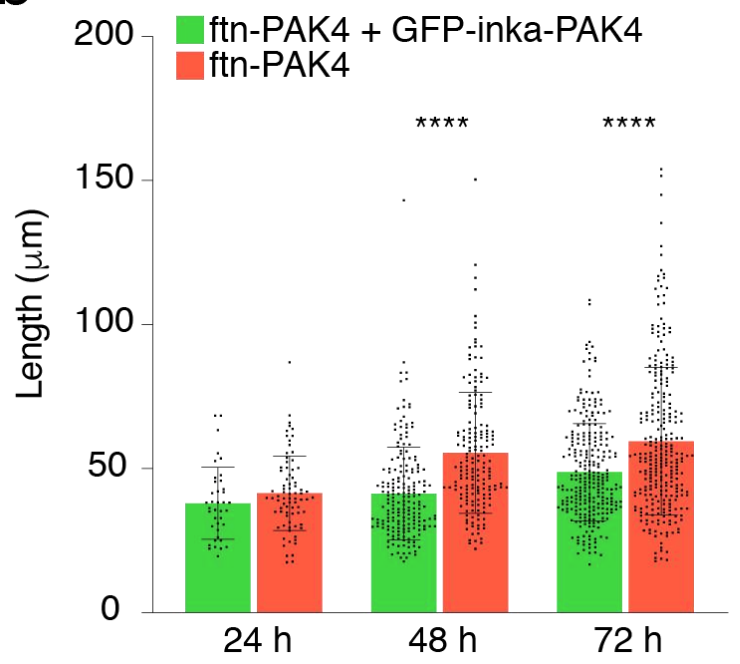

C

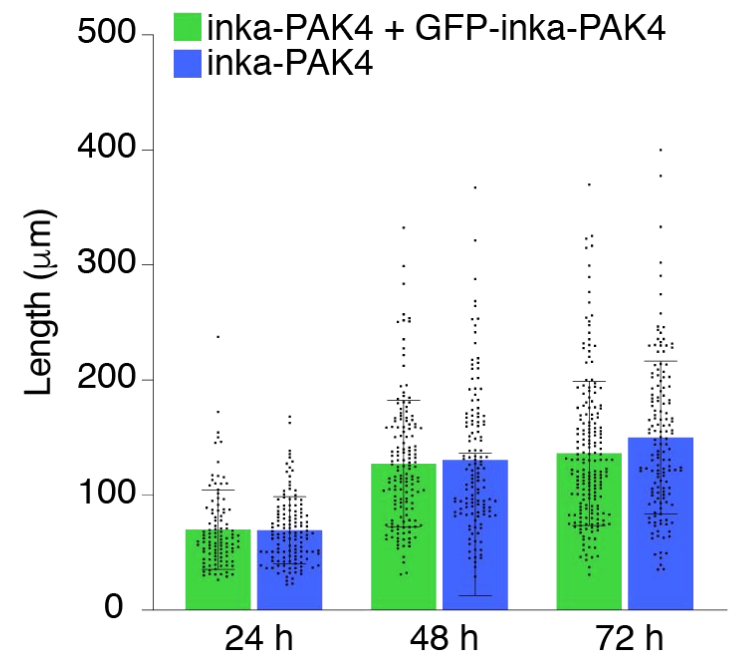

Figure S1. (a) Representative images of HEK293T cells transfected with various ratios of ftnPAK4 and inka-PAK4 plasmids, with GFP-inka-PAK4 added for visibility. (b) Length measurements for inka-PAK4 and GFP-inka-PAK4 crystals over 72 hours. (c) Length measurements for control ftn-PAK4 and control GFP-ftn-PAK4 crystals over 72 hours. 
a

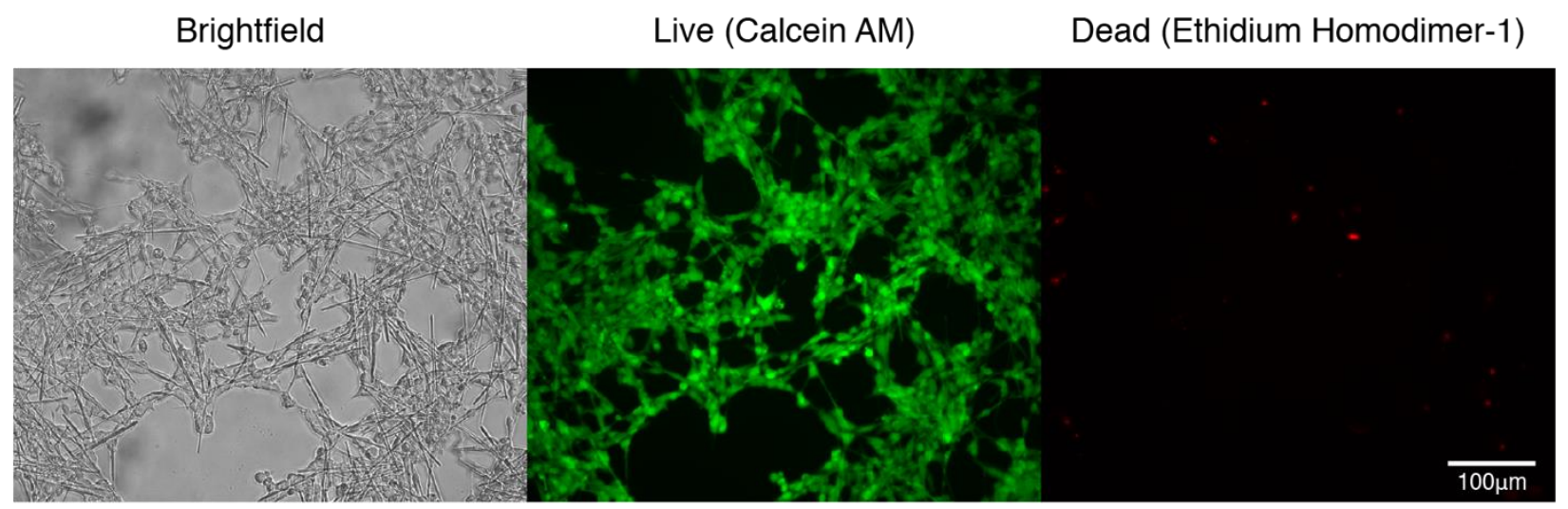

\section{b}

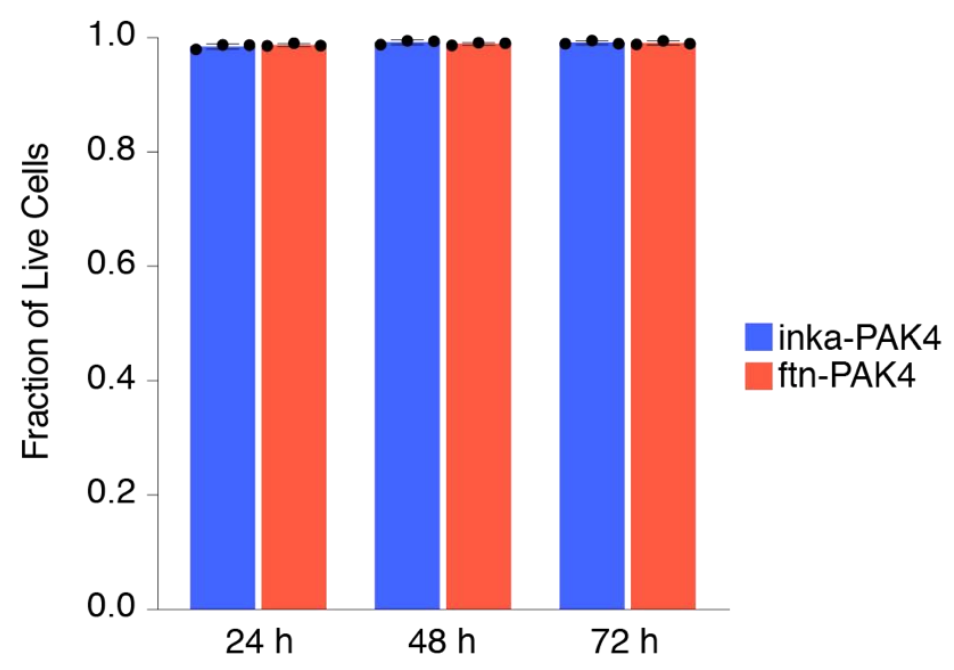

Figure S2. (a) Live/dead staining of HEK cells 72h after transfection with inka-PAK4 (no GFP).

(b) Quantification of cell survival via live/dead staining for HEK cells transfected with ftn-PAK4 and inka-PAK4 over 72h. $\mathrm{n}>700$ cells for each condition. 

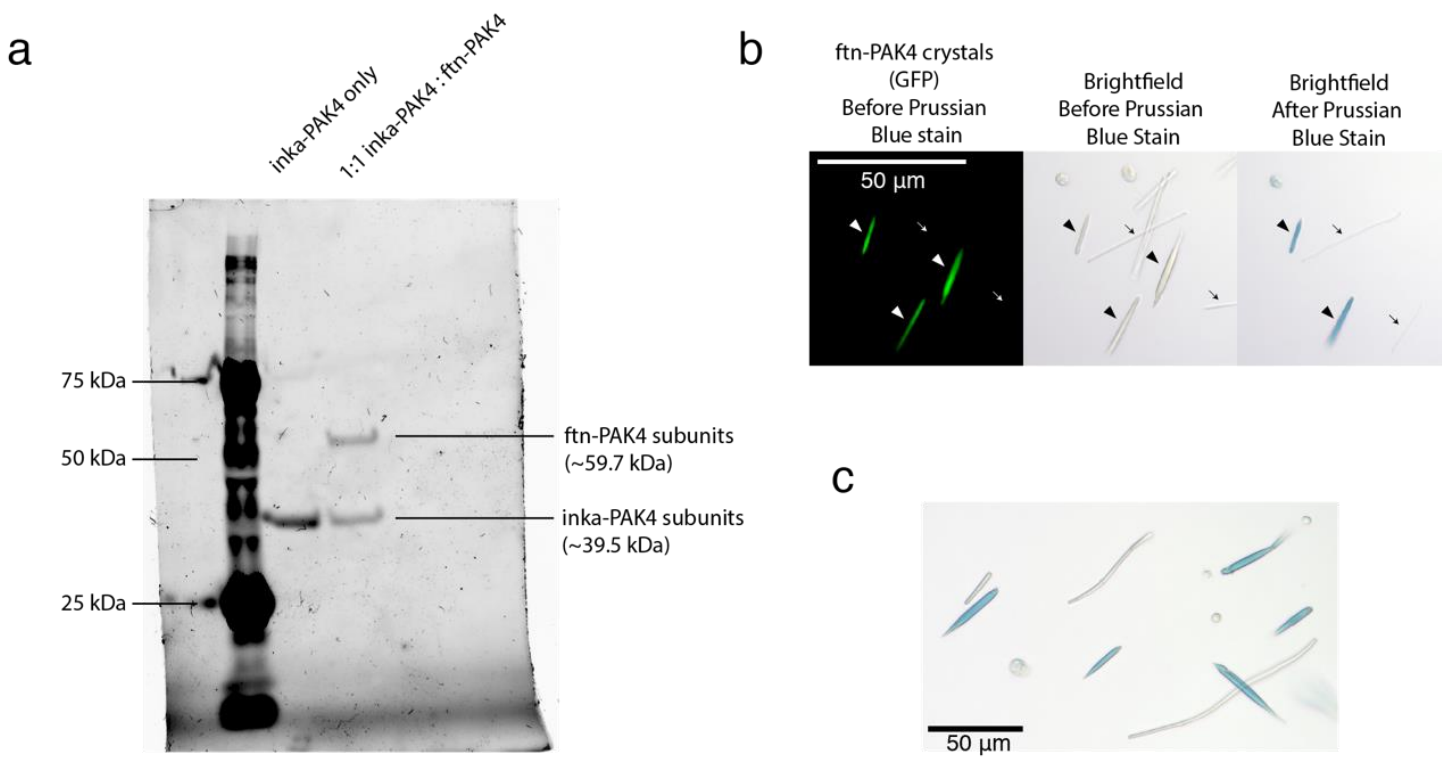

Figure S3. (a) An SDS-PAGE gel of crystals isolated from cells transfected with $100 \%$ inkaPAK4 and 1:1 inka-PAK4 and ftn-PAK4. (b) Images of mixed inka-PAK4 and ftn-PAK4 crystals exposed to iron. In this experiment, the ftn-PAK4 crystals contain GFP-PAK4 for green fluorescence imaging but the control inka-PAK4 crystals do not contain GFP-PAK4. The left and middle images were taken before Prussian Blue staining, and the right image was taken after Prussian Blue staining. Only ftn-PAK4 crystals appear as blue after Prussian Blue staining. The addition of Prussian Blue caused the physical displacement of some crystals, causing them to disappear from the same field of view. The addition of Prussian Blue also quenched the GFP fluorescence. (c) A different field of view of the mixed ftn-PAK4 and inka-PAK4 crystals that had been exposed to iron and stained with Prussian Blue. Only ftn-PAK4 crystals appear as blue after Prussian Blue staining. 
a

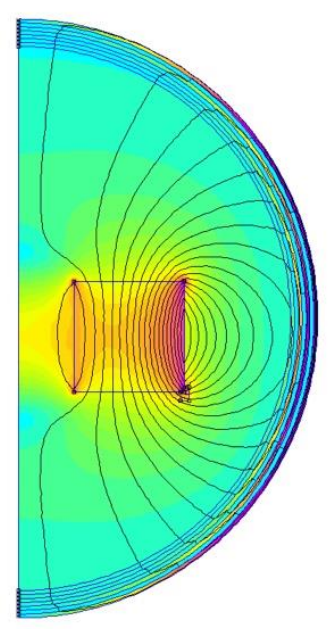

C

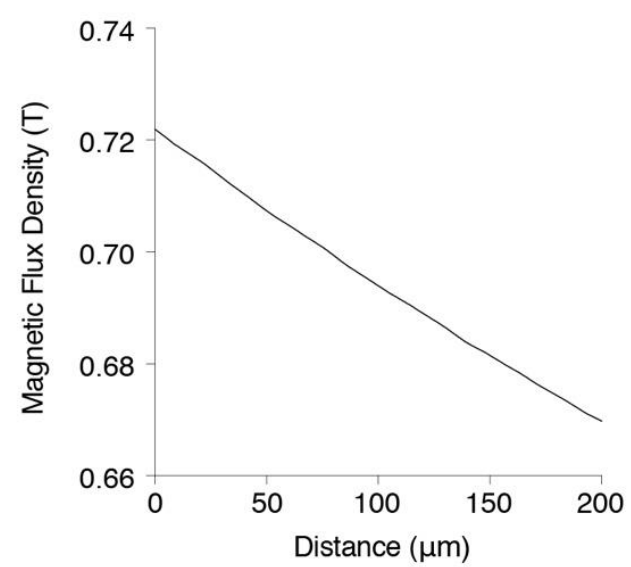

b

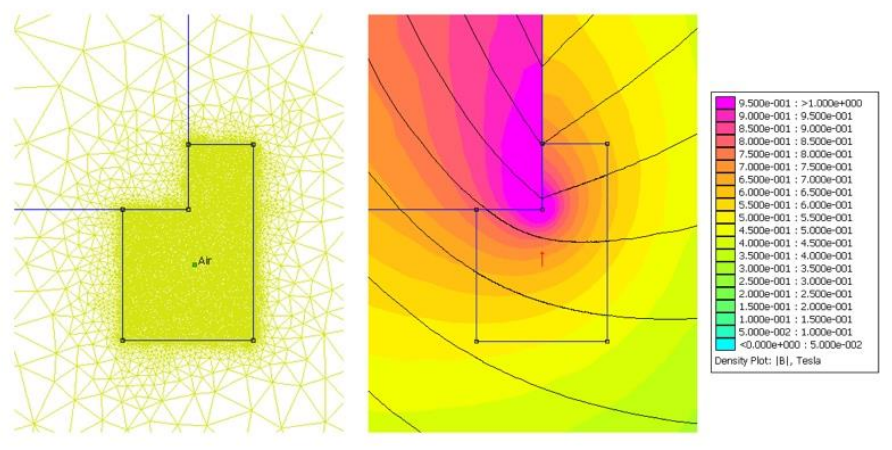

d

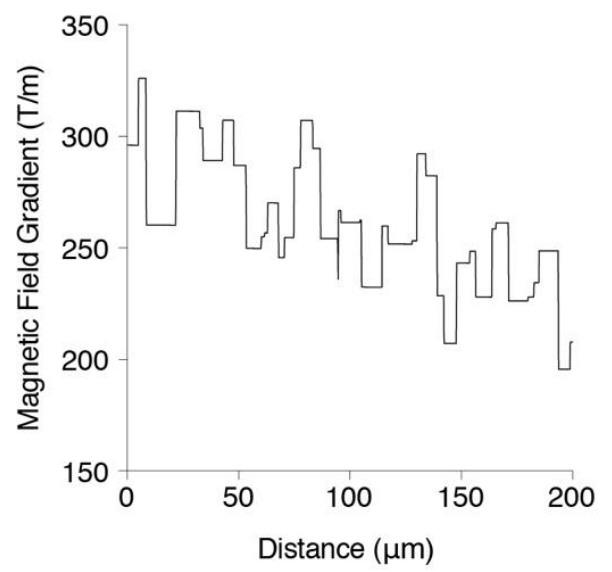

Figure S4. (a) Finite Element Method Magnetics (FEMM) simulation of the magnetic field around a ring magnet. (b) A zoomed-in image of the area under the edge of the magnet, demonstrating the more dense meshing in the region of interest. (c) A plot of the simulated magnetic flux density within the magnetic pulling chamber directly under the permanent magnet. (d) A plot of the magnetic field gradient within the magnetic pulling chamber directly under the permanent magnet. Values were computed by calculating the derivative of the magnetic flux density plot. 

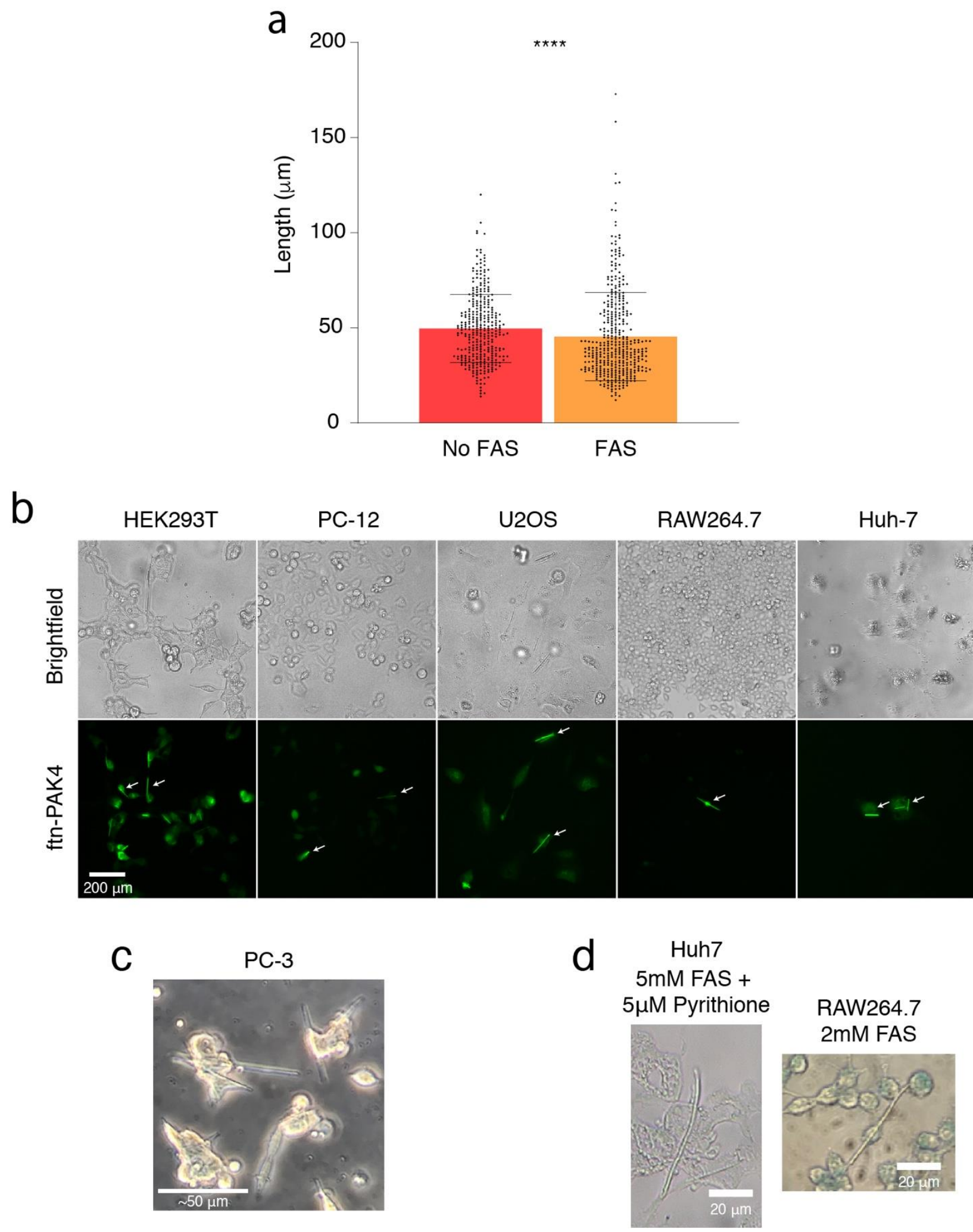

Figure S5. (a) Length measurements of ftn-PAK4 crystals grown in HEK293T cells $72 \mathrm{~h}$ after 
transfection, with and without $5 \mathrm{mM}$ FAS added to the media. For the no FAS condition, $\mathrm{n}=329$ crystals, and for the FAS condition, $\mathrm{n}=401$ crystals, and $\mathrm{p}<0.0001$. (b) ftn-PAK4 crystal assembly $24 \mathrm{~h}$ after transfection in HEK293T, PC-12, U2OS, RAW264.7, and Huh-7 cell lines. White arrows highlight crystal locations. (c) ftn-PAK4 crystal assembly $72 \mathrm{~h}$ after transfection in PC-3 cells. (d) Color imaging of Huh7 and RAW264.7 cells with ftn-PAK4 crystals that have been exposed to iron loading conditions. 
Supplemental Video 1. Time-lapse video of iron-exposed ftn-PAK4 and inka-PAK4 crystals inside the magnetic pulling chamber.

Supplemental Video 2. Time-lapse video of iron-exposed ftn-PAK4 crystals inside the magnetic pulling chamber. During the movie, the magnet is removed and the microscope is re-focused.

Supplemental Video 3. Confocal imaging of iron-exposed ftn-PAK4 and inka-PAK4 crystals inside the magnetic pulling chamber. The top videos are maximum projections of $\mathrm{XZ}$ crosssections of the imaging area, and the bottom videos are maximum projections of the corresponding XY planes.

Supplemental Video 4. Confocal imaging of ftn-PAK4 and inka-PAK4 crystals that have not been exposed to iron inside the magnetic pulling chamber. The top videos are maximum projections of $\mathrm{XZ}$ cross-sections of the imaging area, and the bottom videos are maximum projections of the corresponding XY planes.

Supplemental Video 5. Time-lapse video of HEK293T cells with ftn-PAK4 and inka-PAK4 crystals being pulled by a magnet. Cells are labeled with Cellmask-orange (red), and crystals are GFP (green). 